\title{
Intersection Syndrome: The Subtle Squeak of an Overused Wrist
}

\author{
Thomas M. Skinner, MD
}

Patient histories that include wrist pain can be pivotal in the distinction between intersection syndrome (IS) and the more common de Quervain's tenosynovitis (DQT). Presented here is a 26-year-old pregnant woman with a history of rowing who developed left radial/dorsal wrist pain and a rubbing/squeaking sensation. Nine months of conservative DQT therapy and a landmark-guided corticosteroid injection failed to relieve her symptoms. An in-clinic ultrasound showed tenosynovitis at the intersection of the first and second compartments, confirming a diagnosis of IS. She found immediate relief with ultrasoundguided saline hydrodissection, the injection of saline into the intercompartmental space to reduce adhesions. Both DQT and IS are overuse injuries caused by repetitive wrist extension, as occurs in rowing, and either condition can worsen after pregnancy. Distinguishing the subtleties between DQT and IS can be challenging. Close attention to the patient's description of the pain can guide treatment, potentially expediting recovery. In addition, saline hydrodissection can be both a diagnostic tool and a potentially therapeutic alternative to steroid injections for such tendinopathies. (J Am Board Fam Med 2017;30:547-551.)

Keywords: Cumulative Trauma, de Quervain Disease, Injections, Intersection Syndrome, Pain, Sports Medicine, Wrist Joint

Wrist pain can account for as much as $20 \%$ of musculoskeletal complaints in the ambulatory care setting. ${ }^{1}$ Among the more prevalent diagnoses are tendinopathies of the thumb and wrist, including de Quervain's tenosynovitis (DQT) and intersection syndrome (IS). ${ }^{1}$ While the initial management for both conditions can be similar, understanding their subtle differences may lead to faster recovery and less risk of complications at subsequent visits if initial management should fail. Clues from the patient history can be pivotal in the distinction between IS and the more common DQT. In refractory cases or those in which steroid injections may not be favored, saline hydrodissection (injection of saline into the intercompartmental space to reduce

This article was externally peer reviewed.

Submitted 15 February 2017; revised 29 March 2017; accepted 31 March 2017.

From the US Air Force, 48th Medical Group, Royal Air Force Lakenheath, Suffolk, United Kingdom.

Funding: none.

Conflict of interest: none declared.

Corresponding author: Thomas M. Skinner, MD, 48th Medical Group, Unit 5115, APO, AE, 09464 (E-mail: thomas.skinner@us.af.mil). adhesions) may present a diagnostic and therapeutic alternative.

\section{Case Presentation}

A pregnant 26-year-old woman whose dominant hand is the left hand presented to primary care with a complaint of progressively worsening left wrist pain for the past few weeks. She described a constant, dull ache that had since developed intermittently sharp, shooting pains up her forearm, radiating from a focus just proximal to the metacarpals along the dorsal aspect. She added that there is a "rubbing/friction sensation" over the point of maximum tenderness.

She was 16 weeks into an uncomplicated pregnancy. While in college she was an avid rower, which involved frequent flexion and extension of her left wrist against the resistance of the oar. This dull ache was familiar. However, the sharp shooting pains were different. She had a new hobby baking cupcakes, which required frequently lifting large bags of flour.

Her initial wrist examination revealed no swelling, deformities, or ecchymosis. She had tenderness over the distal aspect of the left radius 2 to $4 \mathrm{~cm}$ 
proximal to the metacarpals along the extensor surface of the forearm. She experienced no pain upon palpation over the metacarpals, the anatomic snuffbox (scaphoid bone), along the first metacarpalphalangeal (MCP) joint, along the rays of the metacarpals, over the distal radial-ulnar junction, or along the boney prominences of the radius or ulna. No palpable mass was felt within the region of the pain or along either the first or second compartments.

She had full range of motion of the left wrist with uninhibited strength when compared with her right side, although ulnar deviation was uncomfortable. She could demonstrate full abduction and adduction of her thumb. Active extension of the thumb against resistance was painful. Ulnar deviation of her wrist while holding her thumb into her palm in a clinched fist, the Finkelstein test (FT), was painful. Her thumb grind test was negative. She had normal capillary refill and normal pinprick sensation along the radial, ulnar, and median nerve regions.

She was suspected to have DQT. Her initial therapy included conservative treatment with trigger avoidance and a thumb-spica splint. She was not a candidate for oral nonsteroidal anti-inflammatory drugs (NSAIDs) because of her pregnancy.

On a subsequent visit, 3 months after delivery, the patient returned to the clinic for a repeat evaluation, reporting that the conservative therapy regimen had not resolved her pain. The shooting pains had decreased in intensity and frequency; however, the constant dull ache persisted. The pain was now most prominent when picking up her 3-month-old child. She developed a new symptom of what she described as a palpable and audible squeaking sensation over the painful region.

Her repeat examination was unchanged with exception that her FT was negative. Still suspecting DQT, she was treated with a landmark-guided corticosteroid injection to the first dorsal compartment. She was asked to start NSAID therapy, continue the thumb-spica splint, and attempt to limit triggers as much as possible.

Two months later, she returned to the clinic, reporting no relief with the splint and steroid injection. Her initial symptoms and the palpable squeak were unchanged, but she had developed a hypopigmented patch and a dimple in her skin along the region of the previous injection. A bedside ultrasound (US) of the intersection of the first and second compartment demonstrated an increased fluid signal along both compartments. She was diagnosed with IS and treated with an USguided saline hydrodissection into the region joining the intersection of the first and second compartments. She experienced immediate relief of her shooting pain and resolution of the palpable/audible squeak.

Her follow-up treatment included scheduled NSAIDs for 2 weeks, rehabilitation at home, and a splint to be worn at night. Two months later she returned for her child's 6-month well visit and reported that her pain had completely resolved and she no longer needed to wear the splint at night.

\section{Discussion}

Wrist pain is a common complaint in ambulatory medicine. ${ }^{1}$ This patient's atraumatic distal forearm pain with an insidious onset and progressive symptoms is most consistent with an overuse injury, matching DQT and IS patterns. ${ }^{2}$

DQT is an inflammation of the tendons in the first dorsal compartment involving the abductor pollicis longus and the extensor pollicis brevis. IS, or "crossover tendonitis," is an inflammation where the tendons of the first compartment cross over the tendons of the second dorsal compartment, extensor carpi radialis longus, and the extensor carpi radialis brevis approximately 2 to $4 \mathrm{~cm}$ proximal to the radial tubercle on the extensor surface of the forearm $^{3}$ (Figure 1).

In both diagnoses, the patient will experience radial/dorsal wrist pain upon heavy lifting and gripping. ${ }^{1}$ However, with IS the pain will be slightly more proximal and dorsal than that demonstrated in DQT (Figure 2). In addition, the patient with IS will typically express a unique friction or "squeaking" sensation in the distal forearm when actively moving the wrist and thumb.

Both diagnoses are associated with overuse injuries in the wrist, caused by repetitive wrist extension such as heavy lifting, rowing, or racket sports. ${ }^{4}$ Both injuries are most common in women 30 to 50 years of age. ${ }^{1,4}$ These injuries can flair up after a pregnancy, also known as "baby wrist," thought to be caused by frequently picking up their child. ${ }^{4-6}$

In either case, the patient will have soft-tissue tenderness along the radial tubercle and lateral portion of the anatomic snuff box. ${ }^{4}$ With DQT, thumb motion will invariably be painful. ${ }^{1}$ Similarly, the 
Figure 1. Anatomic structures involved in de Quervain's tenosynovitis and intersection syndrome.

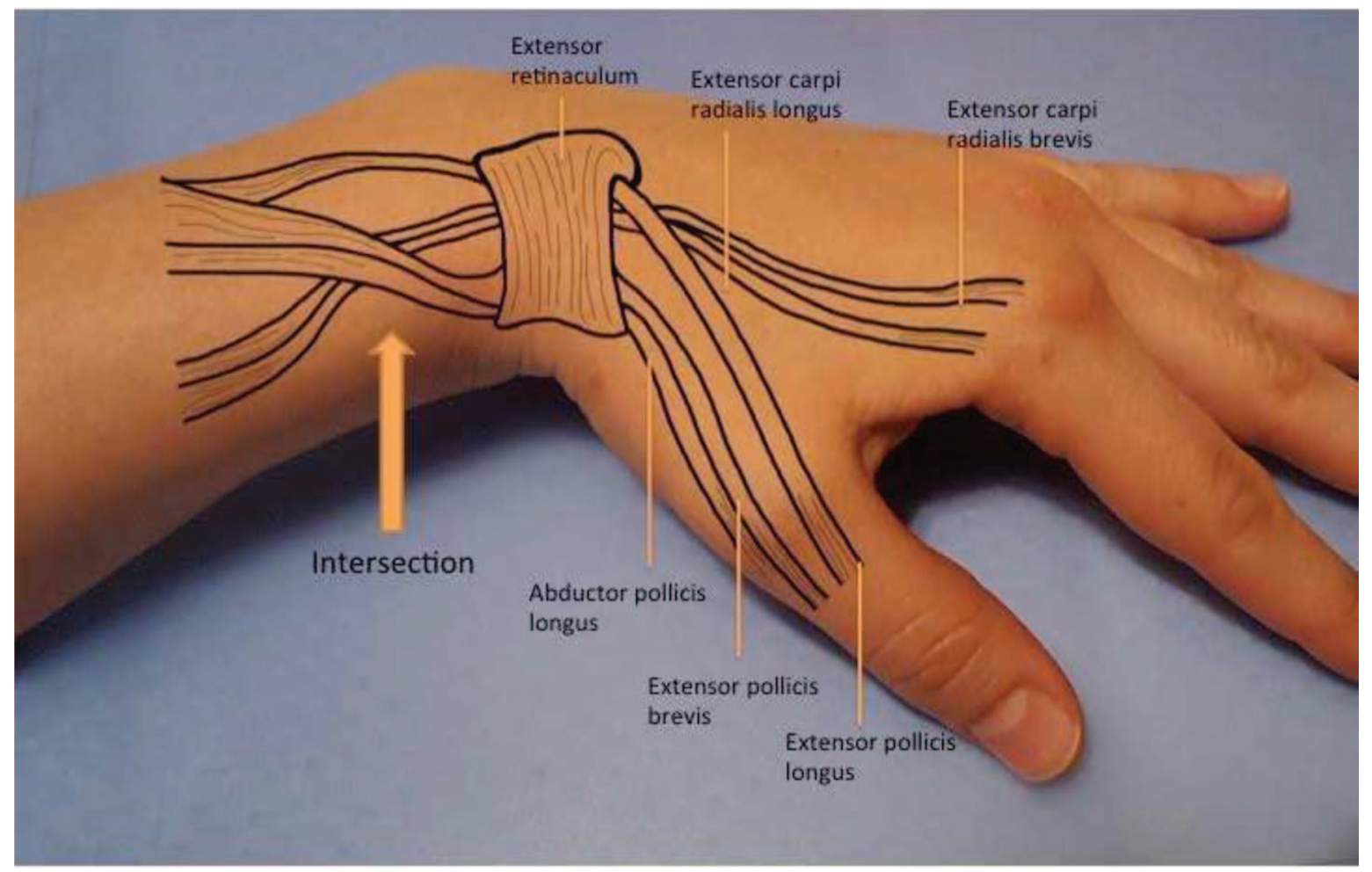

same thumb motion will reproduce pain in patients with IS, although patients with IS often require more prominent movement of the thumb to elicit the pain. In some texts, FT is typically considered a confirmatory test for DQT because of its high sensitivity and specificity (Figure 3 ). ${ }^{7}$ However, other literature has demonstrated that while the test maintains its sensitivity, it lacks specificity in localizing the pain. ${ }^{8}$ Pain from IS, basal MCP joint arthritis, and scaphoid arthritis can each produce a false-positive finding with $\mathrm{FT}^{8}{ }^{8}$ A painless thumb grind maneuver, described as an axial compression

Figure 2. Pain regions unique to intersection syndrome (IS) and de Quervain's tenosynovitis (DQT). IS typically presents with a pain region more proximal and dorsal than that presenting with DQT.
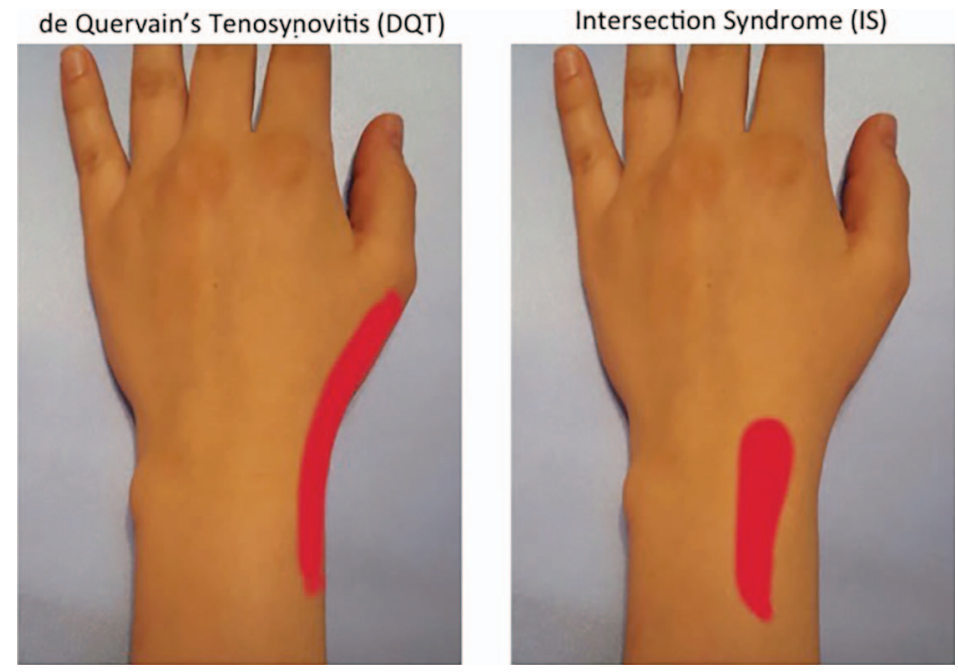
Figure 3. The Finkelstein test stressed the first compartment with a ulnar deviation of the wrist while holding the thumb within the palm.

The Finkelstein's Test

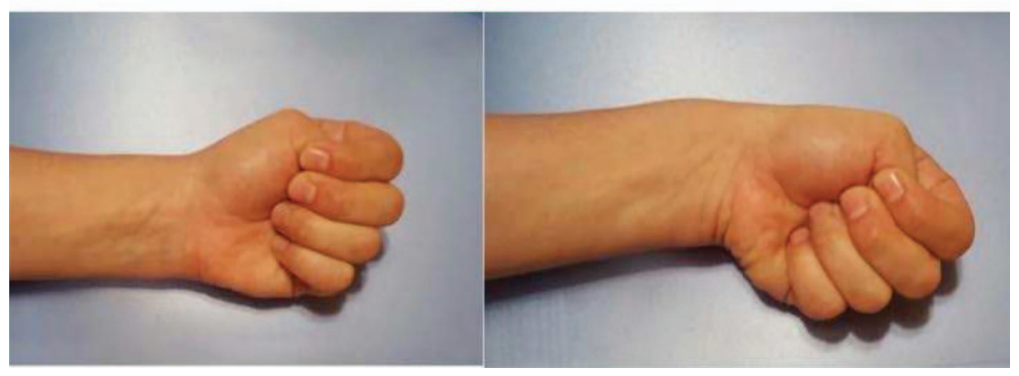

and slight rotation of the MCP joint, will aid in ruling out arthritic pathology. ${ }^{1}$

The use of US is gaining popularity among the Sports Medicine community. ${ }^{9}$ Inflammation of the tendon sheaths can be seen in both DQT and IS; however, US can help localize the inflammation. ${ }^{10}$ US does not require specialized procedures or contrast and therefore has found a place in point-ofcare diagnostics in clinics where a US machine is readily available. ${ }^{9}$

The initial treatment for both DQT and IS is similar to that of many other overuse injuries: antiinflammatories, immobilization when necessary, and rehabilitation. ${ }^{4}$ Although it was not the first treatment tried for this case, moderate evidence (level B) supports using a US-guided steroid injection as first-line therapy for DQT, as it has a higher cure rate over splinting alone (83\% vs $14 \%) .{ }^{11}$ Moreover, this technique is considered safe during pregnancy. ${ }^{11}$

In the short term, steroid therapy has been shown to be more effective than placebo injections or orthotic devices alone. ${ }^{4,12,13}$ However, corticosteroid therapy entails well-known risks. Hypopigmentation and skin atrophy are adverse effects when steroids are applied topically or injected into the dermis. ${ }^{13}$ Tendon rupture is possible with a direct injection of the steroid into the tendon itself. ${ }^{13,14}$ Notably, these risks are generally minimized when the proper steroid type and dosing are injected using US guidance.

Hydrodissection has a growing role in the diagnosis and treatment of tendinopathies. Magnetic resonance imaging evaluation of IS suggests possible entrapment and stenosis of the sheath, ${ }^{15}$ which may explain why hydrodissection worked in this case. Moreover, the technique has been tried with some success with other forms of tendon sheath tethering and contraction. ${ }^{16}$

As a pregnant baker with history of rowing experience, this patient had notable risk factors for developing an overuse injury such as DQT or IS. With a positive FT and the absence of other remarkable physical examination findings, an initial suspicion for DQT was reasonable. Development of a "squeaking" sensation and the location of her pain were both early clues to her final diagnosis of IS. Having failed initial therapy and developed complications to a landmarkguided steroid injection, her diagnosis was eventually confirmed with US evidence of IS.

\section{Conclusion}

Identifying the subtle distinction between a DQT and IS can be challenging. Both diagnoses share similar overuse patterns and aggravating factors. Close attention to the patient's evolution of symptoms can help guide appropriate initial diagnostic and treatment modalities, which may help avoid complications of steroid therapy in the proper setting.

The author thanks Shanna Rodgers for research assistance, editing, and graphic design; and Dr. Antoin "Marcus" Alexander for his assistance with the final diagnosis and management of the patient.

To see this article online, please go to: http://jabfm.org/content/ 30/4/547.full.

\section{References}

1. Ramsey S, Mirabelli MH. Evaluation and diagnosis of wrist pain: a case-based approach. Am Fam Physician 2013;87:568-73.

2. Hanlon DP, Luellen JR. Intersection syndrome: a case report and review of literature. J Emerg Med 1999;17:969-71. 
3. Lee RP, Hatem SF, Recht MP. Extended MRI findings of intersection syndrome. Skeletal Radiol 2009; 38:157-63.

4. Boggess BR. Evaluation of the adult with subacute or chronic wrist pain. Waltham, MA: UpToDate. Last updated March 21, 2017. Available from: https:// www.uptodate.com/contents/evaluation-of-the-adultwith-subacute-or-chronic-wrist-pain. Accessed March 25, 2017.

5. Anderson SE, Steinbach LS, De Monaco D, Bonel HM, Hurtienne Y, Voegelin E. "Baby wrist": MRI of an overuse syndrome in mothers. AJR Am J Roentgenol 2004;182:719-24.

6. Schumacher HR Jr, Dorwart BB, Korzeniowski OM. Occurrence of de Quervain's tendinitis during pregnancy. Arch Intern Med 1985;145:2083-4.

7. Wolf JM, Sturdivant RX, Owens BD. Incidence of de Quervain's tenosynovitis in a young, active population. J Hand Surg Am 2009; 34:112-5.

8. Dawson C, Mudgal CS. Staged description of the Finkelstein test. J Hand Surg Am 2010;35:1513-5.

9. Yim ES, Corrado G. Ultrasound in athletes: emerging techniques in point-of-care practice. Curr Sports Med Rep 2012;11:298-303.
10. Draghi F, Bortolotto C. Intersection syndrome: ultrasound imaging. Skeletal Radiol 2014;43:283-7.

11. Stephens MB, Beutler AI, O’Connor FG. Musculoskeletal injections: a review of the evidence. Am Fam Physician 2008;78:971-6.

12. Coombs BK, Bisset L, Vicenzino B. Efficacy and safety of corticosteroid injections and other injections for management of tendinopathy: a systematic review of randomized controlled trials. Lancet 2010; 376:1751-67.

13. Peters-Veluthamaningal C, Winters JC, Groenier $\mathrm{KH}$, Meyboom-DeJong B. Randomized controlled trial of local corticosteroid injections for de Quervain's tenosynovitis in general practice. BMC Musculoskelet Disord 2009;10:131.

14. Liang J, McElroy K. Hypopigmentation after triamcinolone injection for de Quervain tenosynovitis. Am J Phys Med Rehabil 2013;92:639.

15. Costa CR, Morrison WB, Carrino AJ. Mri features of intersection syndrome of the forearm. AJR Am J Roentgenol 2003;181:1245-9.

16. He L, Genin J, Delzell P. Ultrasound diagnosis and percutaneous treatment of Achilles tendon tethering: a case series. Skeletal Radiol 2016;45:1293-8. 